\title{
The effect of probiotics on depressive symptoms: A meta-analysis of
} randomized controlled trails

\author{
O efeito de probióticos em sintomas depressivos: Uma meta-análise de estudos clínicos \\ randomizados
}

El efecto de los probióticos sobre los síntomas depresivos: Un metanálisis de ensayos controlados aleatorios

\begin{abstract}
Increasing evidence indicates bidirectional communication occurs between the gut and the brain via the microbiomegut-brain axis. Accordingly, several studies point to a positive effect of probiotics on depressive symptomatology. Our study aimed to provide an updated meta-analysis on the subject. We conducted a systematic search for randomized clinical trials in multiple databases. Data analysis was performed using the Review Manager 5.4. We reviewed 26 studies, totaling 2,170 participants. The use of probiotics had a significant effect in reducing depressive symptoms (SMD: - 0.22; 95\% CI: - 0.30 to - 0.13; p < 0.0001) compared to placebo. Subgroup analyses showed that probiotics influenced outcomes in participants with depression (SMD: - 0.67; 95\% CI: - 0.93 to $-0.41 ; p<0.00001$ ) and with other clinical diagnoses (SMD: - 0.23; 95\% CI: - 0.36 to $-0.09 ; \mathrm{p}=0.0008$ ) but not healthy participants (SMD: - 0.11; $95 \%$ CI: -0.23 to $0.02 ; \mathrm{p}=0.100$ ). The use of probiotics can be a promising approach in improving the treatment of depression. It is important to conduct further studies to better assess the efficacy of probiotic as an adjuvant treatment to antidepressant medications, as well as their most effective dosages.
\end{abstract}

Keywords: Probiotics; Depression; Mood disorders; Gastrointestinal microbiome.

\section{Resumo}

Cada vez mais evidências indicam que a comunicação bidirecional ocorre entre o intestino e o cérebro por meio do eixo microbioma-intestino-cérebro. Assim, vários estudos apontam para um efeito positivo dos probióticos na sintomatologia depressiva. Nosso estudo teve como objetivo fornecer uma meta-análise atualizada sobre o assunto. Realizamos uma busca sistemática de ensaios clínicos randomizados em vários bancos de dados. A análise dos dados foi realizada usando o Review Manager 5.4. Revisamos 26 estudos, totalizando 2.170 participantes. O uso de probióticos teve um efeito significativo na redução dos sintomas depressivos (SMD: - 0,22; IC 95\%: - 0,30 a - 0,13; p $<0,0001)$ em comparação com o placebo. As análises de subgrupo mostraram que os probióticos influenciaram os resultados em participantes com depressão (SMD: - 0,67; IC 95\%: - 0,93 a - 0,41; p <0,00001) e com outros diagnósticos clínicos (SMD: - 0,23; IC 95\%: - 0,36 a - 0,09; p =0,0008), mas não participantes saudáveis (SMD: - 
0,11; IC 95\%: - 0,23 a 0,02; $\mathrm{p}=0,100)$. O uso de probióticos pode ser uma abordagem promissora para melhorar o tratamento da depressão. É importante a realização de mais estudos para avaliar melhor a eficácia do probiótico como tratamento adjuvante aos medicamentos antidepressivos, bem como suas dosagens mais eficazes.

Palavras-chave: Probióticos; Depressão; Transtornos do humor; Microbioma gastrointestinal.

\begin{abstract}
Resumen
Cada vez hay más pruebas que indican que se produce una comunicación bidireccional entre el intestino y el cerebro a través del eje microbioma-intestino-cerebro. En consecuencia, varios estudios apuntan a un efecto positivo de los probióticos sobre la sintomatología depresiva. Nuestro estudio tuvo como objetivo proporcionar un metanálisis actualizado sobre el tema. Realizamos una búsqueda sistemática de ensayos clínicos aleatorios en múltiples bases de datos. El análisis de datos se realizó utilizando el Review Manager 5.4. Revisamos 26 estudios, con un total de 2170 participantes. El uso de probióticos tuvo un efecto significativo en la reducción de los síntomas depresivos (DME: 0,22; IC del 95\%: - 0,30 a - 0,13; p <0,0001) en comparación con el placebo. Los análisis de subgrupos mostraron que los probióticos influyeron en los resultados en los participantes con depresión (DME: - 0,67; IC del 95\%: - 0,93 a 0,41; $\mathrm{p}<0,00001$ ) y con otros diagnósticos clínicos (DME: - 0,23; IC del 95\%: - 0,36 a - 0,09; $\mathrm{p}=0,0008$ ) pero no participantes sanos (DME: - 0,11; IC del 95\%: - 0,23 a 0,02; p =0,100). El uso de probióticos puede ser un enfoque prometedor para mejorar el tratamiento de la depresión. Es importante realizar más estudios para evaluar mejor la eficacia de los probióticos como tratamiento adyuvante de los medicamentos antidepresivos, así como sus dosis más efectivas.
\end{abstract}

Palabras clave: Probióticos; Depresión; Trastornos del humor; Microbioma gastrointestinal.

\title{
1. Introduction
}

The human intestine is colonized by approximately $3.8 \times 10^{\wedge} 13$ microorganisms, a number larger than that of actual human cells (Sender et al., 2016). These microorganisms can communicate with the central nervous system (CNS) through several mechanisms. The gut microbes produce $\gamma$-aminobutyric acid (GABA), serotonin and catecholamines. These substances work as local neurotransmitters, modulating exocrine secretion, local motility and vagal afferences, consequently can indirectly stimulates the CNS. Gut microbiota can still produce metabolites such as short-chain fatty acids (SCFA) which can cross the blood-brain barrier and influence neuron's metabolism. Microbes can elicit the production of pro-inflammatory cytokines that influence microglia activation and stimulate the brain through vagus nerve. The CNS, in turn, can modulate the release of substances, intestinal motility and permeability. This set of bidirectional communication is called the microbiota-gut-brain axis (Sherwin et al., 2016).

The intestinal microbiota manipulation in animal models results in visible behavioral changes (Reis et al., 2018). Clinical evidence associates abnormalities of the normal microbiota (dysbiosis) with the appearance of neurological and psychiatric diseases (Kong et al., 2019; Jiang et al., 2015), thus encouraging research that uses microorganisms (probiotics) in the prevention and treatment of these diseases.

In recent years, the clinical use of probiotics has expanded. Meta-analyses have confirmed its effectiveness in the treatment of irritable bowel syndrome (IBS) and ulcerative colitis although the strength of evidence is low and probiotic supplement is not yet broadly recommended (Niu et al., 2020; Astó et al., 2019; Guo et al. 2019).

Besides, new evidence reveals that probiotics have effects on psychiatric symptoms (Sherwin et al., 2016). For example, studies have shown that the consumption of probiotics significantly reduced the perception of depressed mood (Steenbergen et al., 2015), reduced depression scores,10 and improved mental health measures 
in healthy adults (Mohammadi et al., 2016). This opens the possibility that probiotics could be used to treat and prevent psychiatric illnesses and relieve psychological stress.

According to the World Health Organization (WHO, 2017), psychological stress affects $90 \%$ of the world's population and mental disorders are among the most disabling diseases. Major depressive disorder (MDD) is one of the most prevalent psychiatric illnesses. It is characterized by prolonged lowering of mood and may be accompanied by changes in memory, attention, sleep, motivation, muscle tension, and autonomic functioning (Kupfer et al. 2014). Psychiatric diseases have a great social and economic impact, being associated with low school performance and lower productivity at work, a greater number of traffic accidents, drug abuse, and suicide (WHO, 2001).

The pathophysiology of psychic diseases is complex and multifactorial, involving several neural, inflammatory, and metabolic pathways, which makes prevention and treatment difficult. Current therapeutic approaches to depression are not satisfactory, since the average response to the most used drugs is around $50 \%$ (McCormack et al., 2018). Clinical evidence suggests an association between psychic and gastrointestinal symptoms (Ma et al., 2019; Stasi et al., 2019). Abnormalities in the intestinal microbiota, common in psychiatric patients, may compose the pathophysiology of mental illness and/or aggravate the psychic symptoms of individuals in this condition (Petra et al., 2015; Myers et al., 2009).

Depression can be screened and diagnosed using assessment instruments. These instruments are validated scales of either interview or self-reported measurements that quantify depressive symptoms and determine treatment outcomes. Some notorious examples are Beck Depression Inventory (BDI) and Hamilton Depression Rating Scales (HAM-D) (https://www.apa.org/depression-guideline/assessment)

The relevance of mental illness in our society, the limitations of usual treatment, and the large availability of probiotics in the market justify the review of the evidence obtained in randomized controlled trials (RCT) on the effect of probiotics on depressive symptoms. This study aimed to examine the effect of probiotics on depression scales and to separately analyze subgroups of participants.

\section{Methods}

The literature search was carried out according to the Preferred Reporting Items for Systematic Reviews and Meta-Analyses (PRISMA) (Liberati et al., 2009; Moher et al 2009). It was registered in the International Prospective Register of Systematic Reviews (PROSPERO) with ID number 213949.

\section{Search Strategy}

The systematic review was carried out by consulting the PubMed, ScienceDirect, LILACS, and SciELO databases, from the oldest records until those published in June 2021. The search strategy was initially developed for PubMed and was later modified to suit the interface of other databases. The keywords were the health descriptors available in DECS and MeSH. The basic research strategy included [Probiotics OR Gastrointestinal Microbiome OR Lactobacillus OR Bifidobacterium OR Bacteria] AND [Anxiety OR Mood OR Stress OR depression OR Mental Health]. 
There was no language restriction. An initial screening of titles and abstracts identified potential studies, after which an analysis of complete articles was conducted to definitively include or exclude the study based on the eligibility criteria described below. This process was carried out independently by two of the authors (VP and MP). Any disagreement was resolved through discussion and consensus. In cases where consensus could not be reached, it was planned that a third author (CG) would make the final decision.

\section{Eligibility Criteria and Study Selection}

The inclusion criteria were the following: (1) RCT design comparing probiotic and placebo; (2) Studies in healthy humans or with a diagnosed psychiatric illness, such as depression or anxiety; (3) Any formulation and administration of probiotics that report their values of colony-forming units (CFU), species of the product, dosages, and duration of the intervention; (4) Use of validated scales for symptoms of depression; (5) Studies containing pre- and postintervention results in the form of means and standard deviations, or that could be converted mathematically into these two measures.

Studies that used probiotics in addition to some other nutrient or adjuvant, such as vitamin D or prebiotics, were included. Studies were also included in which the volunteers had other diseases such as fibromyalgia (Roman et al., 2018) and polycystic ovary syndrome (Jamilian et al., 2018; Ostadmohammadi et al. 2019).

Studies with the respective criteria were excluded from the meta-analysis: (1) Use of probiotics to treat gastrointestinal diseases (IBS, ulcerative colitis, antibiotic-associated diarrhea), considering that probiotics may have a positive effect on the symptoms of disease and secondarily impact mood and depression scales; (2) They did not provide clear or calculable data before and after intervention or incomplete data; (3) Inclusion of children or adolescents ( $\leq 18$ years); (4) Studies exclusively reported anxiety measures; (5) Instruments related to stress, quality of sleep, quality of life, general health condition, and intestinal symptoms were excluded from the metaanalysis to reduce the heterogeneity of the studies.

The search methodology was outlined in a flowchart and described in Figure 1.

\section{Data Extraction}

The relevant data were extracted using the Google Forms tool and later transferred to an Excel spreadsheet. A standard table was developed by author VP, MP and CG following the Cochrane Handbook for Systematic Reviews of Interventions.

For the articles that made their results available in standard error of mean (SEM), we transformed them into standard deviations (SD) using the mathematical formula $\mathrm{SEM}=\mathrm{SD} / \sqrt{ }$, for which " $\mathrm{n}$ " represents the size of the sample studied.

Dropout values were compared between the placebo and probiotic groups to assess the impact of adverse effects on the study segment. 


\section{Risk of Bias Assessment}

The risk of bias was measured using the Cochrane Risk of Bias Assessment Tool (Higgins et al., 2011) and is described in Figure 2.

\section{Statistical Analysis}

Each study was conducted separately by two authors and analyzed using Revman 5.4 software. The treatment effect was calculated using standardized mean difference (SMD) between the value of the first postintervention measure in the probiotic group and the value in the placebo group for the depression symptom scales. This methodology was used both for the total analysis and for the analysis of subgroups.

Heterogeneity between studies was assessed using the chi-square (X2) test and I2 statistic. Great heterogeneity between articles is indicated when $\mathrm{p}<0.05$ for $\mathrm{X} 2$ or $\mathrm{I} 2>50 \%$. Possible publication bias was investigated using a funnel plot (Egger et al., 1997).

We proceeded with the analysis of three subgroups: 1) MDD patients; 2) participants with clinical diagnoses other than depression; 3) healthy participants.

A t-test for independent samples was performed to investigate the difference between mean dropout rates of the placebo and probiotic groups.

\section{Results}

\section{Included Studies}

From a total of 2,185 studies, we analyzed 26 double-blinded, randomized, placebo-controlled trials. Figure 1 shows the identification, screening, selection criteria, and inclusion process.

Twelve studies investigated the effect of probiotics in healthy patients (Chung et al., 2014; Lee et al., 2021; Messaoudi et al., 2011; Mohammadi et al., 2016; Nishida et al., 2019; Nishihira et al., 2014; ÖstlundLagerström et al., 2016; Romijn et al., 2017; Siegel \& Conklin, 2020; Smith-ryan et al., n.d.; Steenbergen et al., 2015; Venkataraman et al., 2021). In terms of clinical diagnoses, five studies used a sample of patients diagnosed with MDD (Akkasheh et al., 2016; Chahwan et al., 2019; Ghorbani et al., 2018; Kazemi et al., 2019; Reininghaus et al., 2020), two studies included women with polycystic ovarian syndrome (POS) (Jamilian et al., 2018; Ostadmohammadi et al., 2019) two studies integrated patients with type 2 diabetes mellitus and coronary heart disease (Raygan et al., 2018, 2019), two studies included a sample of pregnant women (Dawe et al., 2020; Slykerman et al., 2017), one study comprised patients with myocardial infarction who underwent percutaneous coronary intervention,(Moludi et al., 2019) one article included patients with coronary artery disease (CAD) (Moludi et al., 2021) and one study included patients with fibromyalgia,(Roman et al., 2018). The former nine articles made up the "other clinical diagnoses" subgroup. 
Figure 1: PRISMA flow diagram of studies selected.

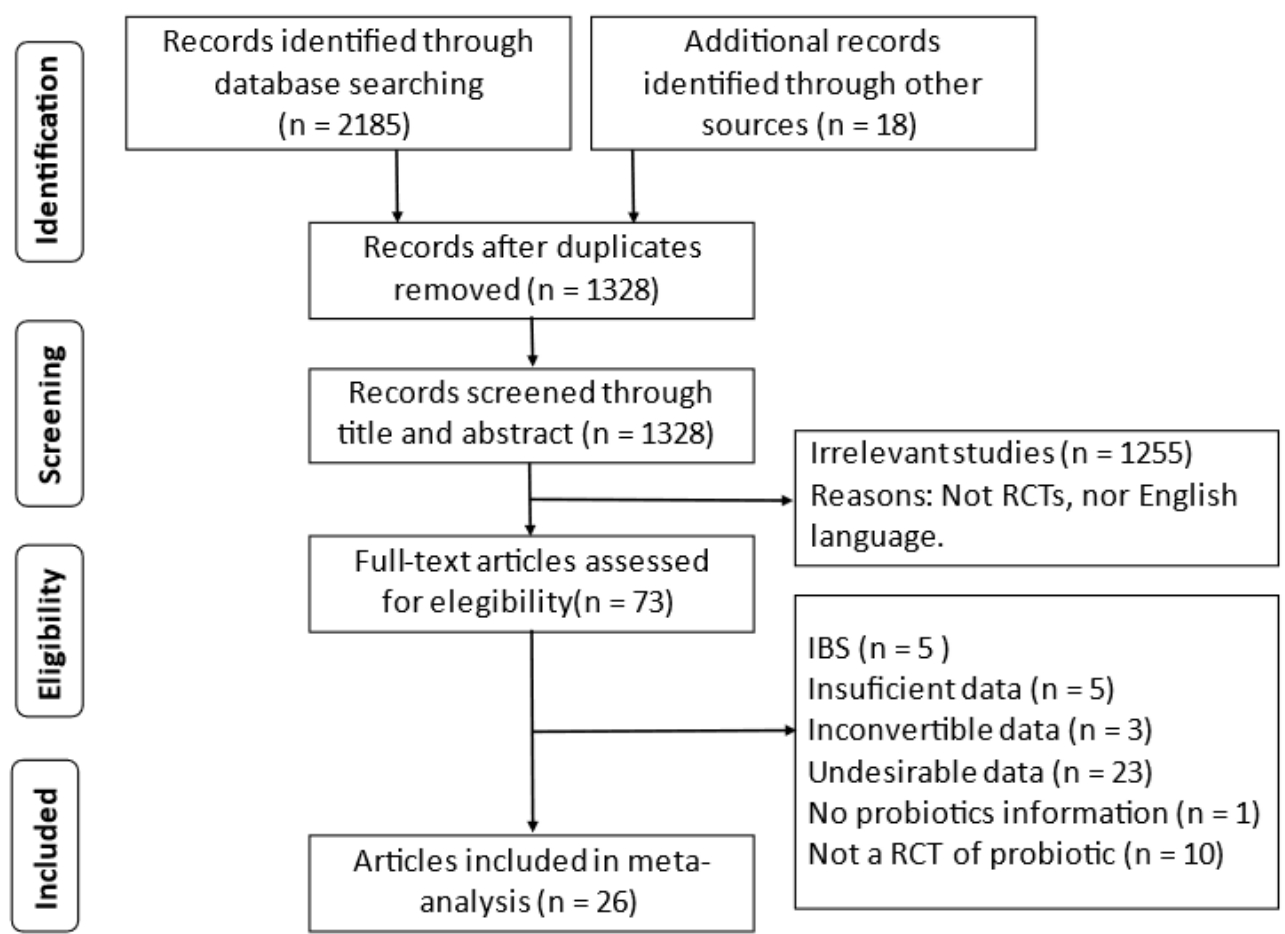

Irritable Bowel Syndrome (IBS); Randomized Controlled Trial (RCT).

Source: Palmares et al., (2021).

\section{Quality Assessment}

Figure 2A summarizes the risk of bias for each study. Figure 2B presents the risk of bias of all RCTs included in the study using a distribution chart of the reviewers' judgments. Eight studies showed a high risk of bias for a single domain, but the overall risk of bias was low. 
Figure 2: (A) Risk of bias for each RCT included low risk of bias (+), high risk of bias (-), and unclear risk of bias (?). (B) Bar chart comparing the percent risk of bias for each RCT included.
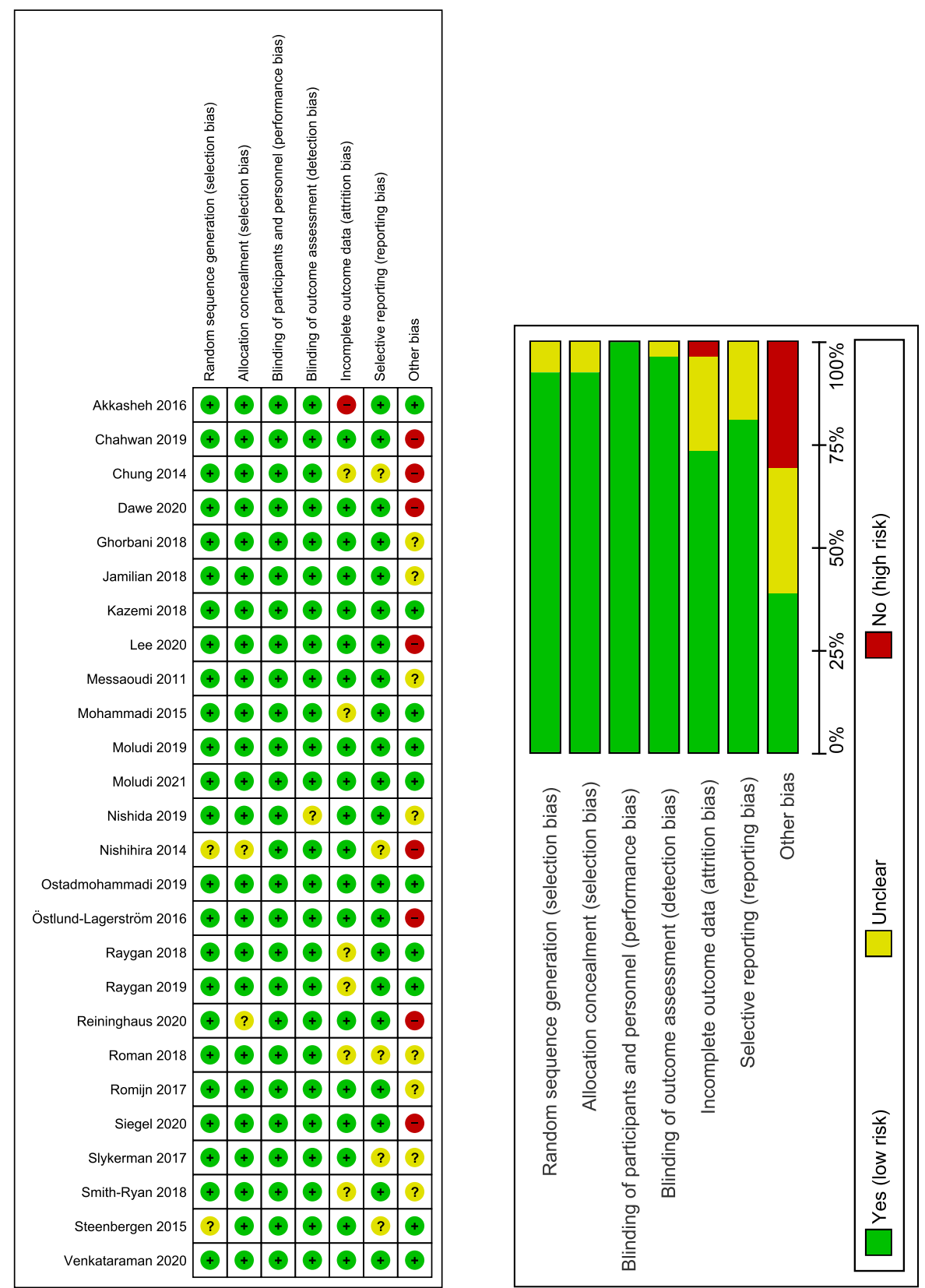

Source: Palmares et al., (2021).

\section{Probiotics and Depression}

Main characteristics of the 26 RCTs are summarized in Table 1, which contains summary information about the date of publication, country of study, general condition of participants, number of patients recruited, and the average age for each intervention group: probiotic (PRO) or placebo (PLA). Further, the gender of participants, species, and strain of the probiotic, dosage in CFU total daily, presentation of the probiotic, duration of the intervention, and results of interest to this meta-analysis are also summarized. 
We reviewed data on 2,170 human subjects (intervention group $=1099$; control group $=1071$ ). The effectiveness of the use of probiotics in depressive symptoms is summarized in Figure 3. Significant improvement was found in depressive symptoms in the probiotic group (SMD: $-0.22 ; 95 \%$ CI: -0.30 to $-0.13 ; \mathrm{p}<0.0001 ; \mathrm{I}^{2}=$ $78 \%$ ) when considering the first postintervention measure of all 26 studies in the analysis.

\section{Subgroup Analysis of Probiotic Efficacy Based on Clinical Condition}

Assessing the efficacy of a probiotic over a placebo for the subgroup of patients diagnosed with MDD involved five studies, showing a significant impact on reducing depressive symptoms in the first postintervention measure (SMD: - 0.67; 95\% CI: -0.93 to $-0.41 ; \mathrm{p}<0.00001 ; \mathrm{I}^{2}=74 \%$ ). For participants with other clinical diagnoses, a significant improvement was also found compared to a placebo (SMD: - 0.23; 95\% CI: - 0.36 to $0.09 ; \mathrm{p}=0.0008 ; \mathrm{I}^{2}=32 \%$ ). As for healthy participants, treatment with probiotics did not have a significant effect (SMD: - 0.11; 95\% CI: -0.23 to $0.02 ; \mathrm{p}=0.100 ; \mathrm{I}^{2}=84 \%$ ). The results are summarized in Figure 3 . The general analysis of all studies obtained $\mathrm{I}^{2}=78 \%$, considerable heterogeneity, while the analyses of the subgroups showed $\mathrm{I}^{2} \geq 32 \%-84 \%$, moderate to substantial heterogeneity.

\section{Publication Bias and Sensitivity Analyses}

Regarding the funnel plot (Figure 4), the visual inspection of the figure showed an asymmetric distribution of the studies. This suggests a possible bias of publishing that favors studies reporting a positive effect of probiotics.

The results of the sensitivity analysis did not show significant changes between groups. This test was performed excluding each study individually, including those most vulnerable to bias.

\section{Adverse Effect of Probiotics}

The number of volunteers who discontinued treatment was slightly greater in the probiotic group when considering all 26 studies. The independent student's t-test showed no significant difference between the means of dropping out of the placebo and probiotic groups $(\mathrm{p}=0.791)$. 
Table 1: Characteristics of included studies

\begin{tabular}{|c|c|c|c|c|c|c|c|c|c|}
\hline $\begin{array}{l}\text { Study (year) } \\
\text { location }\end{array}$ & Population Condition & $\mathbf{N}$ & $\begin{array}{l}\text { Sex rate } \\
(\text { male } \%)\end{array}$ & Age $(\operatorname{mean} \pm$ SD $)$ & Probiotic strain (CFU/day) & Design & Duration & $\begin{array}{l}\text { Outcome } \\
\text { Measure }\end{array}$ & Results \\
\hline $\begin{array}{l}\text { Akkasheh et al. } \\
\text { (2016) Iran }\end{array}$ & Patients with MDD & $\begin{array}{l}\text { PRO: } \\
\text { PLA: } 20\end{array}$ & $15 \%$ & $\begin{array}{l}\text { PRO: } 38.3 \pm 12.1 \\
\text { PLA: } 36.2 \pm 8.2\end{array}$ & $\begin{array}{l}\text { Lactobacillus acidophilus }(2 \mathrm{x} \\
\left.10^{9}\right), \text { L. casei }\left(2 \times 10^{9}\right), \text { B. bifidum } \\
\left(2 \times 10^{9}\right)\end{array}$ & Capsule & 8 weeks & BDI & PRO $>$ PLA $* *$ \\
\hline $\begin{array}{l}\text { Chung et al. } \\
(2014) \\
\text { South Korea }\end{array}$ & Healthy older adults & $\begin{array}{l}\text { PRO: } \\
\text { PLA: } 10\end{array}$ & $47.4 \%$ & $\begin{array}{l}\text { PRO: } 66.6 \pm 5.0 \\
\text { PLA: } 64.5 \pm 4.5\end{array}$ & L. helveticus (CFU not described) & $\begin{array}{l}\text { Fermented } \\
\text { milk }\end{array}$ & 12 weeks & GDS-SF & $\begin{array}{l}\text { NO significant } \\
\text { differences. }\end{array}$ \\
\hline $\begin{array}{l}\text { Ghorbani et al. } \\
\text { (2018) Iran }\end{array}$ & $\begin{array}{l}\text { Patients with MDD treated } \\
\text { with fluoxetine } 20 \mathrm{mg} / \mathrm{d}\end{array}$ & $\begin{array}{l}\text { PRO: } 20 \\
\text { PLA: } 20\end{array}$ & $30 \%$ & $\begin{array}{l}\text { PRO: } 34.5 \pm 4.0 \\
\text { PLA: } 35.5 \pm 5.3\end{array}$ & $\begin{array}{l}\text { Familact } \mathrm{H}{ }^{\circledR} \text { L. casei }\left(3 \times 10^{8}\right), L . \\
\text { acidophilus }\left(2 \times 10^{8}\right), \quad L . \\
\text { bulgaricus }\left(2 \times 10^{9}\right) \text {, B. breve }(2 \times \\
\left.10^{8}\right), \text { B. longun }\left(1 \times 10^{9}\right), \\
\text { Streptococcus termophilus }(3 \times \\
\left.10^{8}\right)\end{array}$ & Capsule & 6 weeks & HDRS & PRO $>$ PLA. \\
\hline $\begin{array}{l}\text { Kazemi et al. } \\
\text { (2019) Iran }\end{array}$ & $\begin{array}{l}\text { Patients with MDD treated } \\
\text { with antidepressants } \\
\text { for } 3 \text { months or more prior to } \\
\text { beginning the trial) }\end{array}$ & $\begin{array}{l}\text { PRO: } \quad 38 \\
\text { PLA: } 36\end{array}$ & $27 \%$ & $\begin{array}{l}\text { PRO: } 36.2 \pm 7.9 \\
\text { PLA: } 36.0 \pm 8.5\end{array}$ & $\begin{array}{l}\text { L. helveticus R0052, B. longum } \\
\text { R0175 }\end{array}$ & Sachet & 8 weeks & BDI & PRO > PLA. \\
\hline $\begin{array}{l}\text { Messaoudi } \text { et al. } \\
\text { (2011) France }\end{array}$ & Healthy adults & $\begin{array}{l}\text { PRO: } 2 \\
\text { PLA: } 29\end{array}$ & $25.5 \%$ & $\begin{array}{l}\text { PRO: } 42.4 \pm 7.5 \\
\text { PLA: } 43.2 \pm 8.5\end{array}$ & $\begin{array}{l}\text { ProbioStick }{ }^{\circledR} \text { L. helveticus } \\
\text { R0052, B. longum R0175 }(3 \mathrm{x} \\
\left.10^{9}\right)\end{array}$ & $\begin{array}{l}\text { Sachet } \\
\text { (powder) }\end{array}$ & 30 days & HADS-D & $\begin{array}{l}\text { NO significant } \\
\text { difference. }\end{array}$ \\
\hline
\end{tabular}




\begin{tabular}{|c|c|c|c|c|c|c|c|c|c|}
\hline $\begin{array}{l}\text { Mohammadi et } \\
\text { al. (2016) Iran }\end{array}$ & $\begin{array}{l}\text { Healthy petrochemical } \\
\text { workers }\end{array}$ & $\begin{array}{l}\text { PRO: } 25 \\
\text { PLA: } 20\end{array}$ & $53.3 \%$ & $\begin{array}{l}\text { PRO: } 31.5 \quad \pm \quad 5.8 \\
\text { PLA: } 33.1 \pm 6.1\end{array}$ & $\begin{array}{l}\text { L. casei }\left(3 \times 10^{3}\right) \text {, L. acidophilus } \\
\left(3 \times 10^{7}\right) \text {, L. rhamnosus }\left(7 \times 10^{9}\right) \text {, } \\
\text { L. bulgaricus }\left(5 \times 10^{8}\right) \text {, B. breve } \\
\left(2 \times 10^{10}\right) \text {, B. longum } \\
\left(1 \times 10^{9}\right) \text {, S. thermophilus }(3 \times \\
\left.10^{8}\right)\end{array}$ & Capsule & 6 weeks & DASS & $\begin{array}{l}\text { PRO > PLA for } \\
\text { DASS. }\end{array}$ \\
\hline $\begin{array}{l}\text { Nishida et al. } \\
\text { (2019) Japan }\end{array}$ & $\begin{array}{l}\text { Healthy students facing } \\
\text { examination stress }\end{array}$ & $\begin{array}{l}\text { PRO: } \quad 31 \\
\text { PLA: } 29\end{array}$ & $59.4 \%$ & $\begin{array}{l}\text { PRO: } 24.9 \pm 2.78 \\
\text { PLA: } 25.3 \pm 3.23\end{array}$ & L. gasseri CP2305 $\left(1 \times 10^{10}\right)$ & Tablet & 24 weeks & HADS-D & $\begin{array}{l}\text { NO significant } \\
\text { difference. }\end{array}$ \\
\hline $\begin{array}{l}\text { Nishihira et al. } \\
\text { (2014) Japan }\end{array}$ & Healthy adults & $\begin{array}{l}\text { PRO: } 115 \\
\text { PLA: } 109\end{array}$ & $30.8 \%$ & $\begin{array}{l}\text { PRO: } 53.6 \pm 11.3 \\
\text { PLA: } 54.3 \pm 10.9\end{array}$ & $\begin{array}{l}\text { L. gasseri SBT } 2055\left(\geq 5 \times 10^{8}\right) \text {, } \\
\text { B. logum SBT } 2928\left(\geq 1 \times 10^{9}\right), S . \\
\text { thermophilus, L. delbrueckii }\end{array}$ & Yogurt & 12 weeks & GHQ-28 & $\begin{array}{l}\text { NO significant } \\
\text { difference. }\end{array}$ \\
\hline $\begin{array}{l}\text { Ostadmohamadi } \\
\text { et al. (2019) } \\
\text { Iran }\end{array}$ & Women with POS & $\begin{array}{l}\text { PRO: } 30 \\
\text { PLA: } 30\end{array}$ & $0 \%$ & $\begin{array}{l}\text { PRO: } 24.4 \quad \pm \quad 4.7 \\
\text { PLA: } 25.4 \pm 5.1\end{array}$ & $\begin{array}{l}\text { L. acidophilus, B. bifidum, } L . \\
\text { reuteri, L. fermentum }\left(2 \times 10^{10}\right. \\
\text { each })+50,000 \text { IU vitamin D }\end{array}$ & Capsule & 12 weeks & BDI & PRO $>$ PLA. \\
\hline $\begin{array}{l}\text { Raygan et al. } \\
\text { (2019) Iran }\end{array}$ & $\begin{array}{l}\text { Patients with type } 2 \text { diabetes } \\
\text { mellitus (DM) and coronary } \\
\text { heart disease (CHD) }\end{array}$ & $\begin{array}{l}\text { PRO: } 27 \\
\text { PLA: } 27\end{array}$ & $38.8 \%$ & $\begin{array}{l}\text { PRO: } 64.8 \quad \pm \quad 8.3 \\
\text { PLA: } 62.4 \pm 13.1\end{array}$ & $\begin{array}{l}\text { L. acidophilus, L. reuteri, } L \text {. } \\
\text { fermentum, B. bifidum }\left(2 \times 10^{9}\right. \\
\text { each })+ \text { selenium }(200 \mu \mathrm{g})\end{array}$ & Capsule & 12 weeks & BDI & PRO > PLA. \\
\hline $\begin{array}{l}\text { Raygan et al. } \\
\text { (2018) Iran }\end{array}$ & $\begin{array}{l}\text { Patients with type } 2 \mathrm{DM} \text { and } \\
\text { CHD }\end{array}$ & $\begin{array}{l}\text { PRO: } \quad 30 \\
\text { PLA: } 30\end{array}$ & $50 \%$ & $\begin{array}{l}\text { PRO: } 71.5 \pm 10.9 \\
\text { PLA: } 67.3 \pm 11.0\end{array}$ & $\begin{array}{l}\text { L. acidophilus, B. bifidum, } L . \\
\text { reuteri, L. fermentum }\left(2 \times 10^{10}\right. \\
\text { each })+50,000 \text { IU vitamin D }\end{array}$ & Capsule & 12 weeks & BDI & PRO > PLA. \\
\hline $\begin{array}{l}\text { Roman et al. } \\
\text { (2018) Spain }\end{array}$ & Patients with fibromyalgia & $\begin{array}{l}\text { PRO: } 16 \\
\text { PLA: } 15\end{array}$ & $10 \%$ & $\begin{array}{l}\text { PRO: } 55 \quad \pm \quad 2.1 \\
\text { PLA: } 50.3 \pm 2.0\end{array}$ & $\begin{array}{l}\text { L. rhamnosus } G G, \text { L. casei, } L . \\
\text { acidophilus, B. bifidus }(2 \mathrm{x})\end{array}$ & Capsule & 8 weeks & BDI & $\begin{array}{l}\text { NO significant } \\
\text { difference. }\end{array}$ \\
\hline $\begin{array}{lr}\text { Romijn } & \text { et al. } \\
(2017) & \text { New } \\
\text { Zealand } & \end{array}$ & $\begin{array}{l}\text { Patients with moderate } \\
\text { depressive symptoms, with } \\
\text { no treatment in the last } 4 \\
\text { weeks }\end{array}$ & $\begin{array}{l}\text { PRO: } \quad 40 \\
\text { PLA: } 39\end{array}$ & $21.5 \%$ & $\begin{array}{l}\text { PRO: } 35.8 \pm 14.0 \\
\text { PLA: } 35.1 \pm 14.5\end{array}$ & $\begin{array}{l}\text { L. helveticus R0052, B. longum } \\
\mathrm{R} 0175\left(\geq 3 \times 10^{9}\right)\end{array}$ & $\begin{array}{l}\text { Sachet } \\
\text { (powder) }\end{array}$ & 8 weeks & QIDS SR16 & $\begin{array}{l}\text { NO significant } \\
\text { difference. }\end{array}$ \\
\hline
\end{tabular}


Research, Society and Development, v. 10, n. 9, e54510918359, 2021

(CC BY 4.0) | ISSN 2525-3409 | DOI: http://dx.doi.org/10.33448/rsd-v10i9.18359

\begin{tabular}{|c|c|c|c|c|c|c|c|c|c|}
\hline Zealand & weeks gestation & PLA: 187 & & PLA: $33.7 \pm 4.4$ & & & & & \\
\hline $\begin{array}{l}\text { Smith-Ryan et } \\
\text { al. (2018) USA }\end{array}$ & $\begin{array}{l}\text { Healthy women } \\
\text { occupational shiftwork }\end{array}$ & $\begin{array}{l}\text { PRO: } 15 \\
\text { PLA: } 18\end{array}$ & $0 \%$ & $\begin{array}{l}\text { PRO: } \quad 30.5 \quad \pm \quad 7.7 \\
\text { PLA: } 30.2 \pm 10.0\end{array}$ & $\begin{array}{l}\text { Ecologic }{ }^{\circledR} \text { Barrier: B.bifidum } \\
\text { W23, B. lactis W51, B. lactis } \\
\text { W52, L. acidophilus } \\
\text { W37, L. brevis W63, L. casei } \\
\text { W56, L. salivarius W24, L. } \\
\text { lactis }\left(\text { W19 and W58), }\left(2.5 \times 10^{9}\right)\right.\end{array}$ & $\begin{array}{l}\text { Sachet } \\
\text { (powder) }\end{array}$ & 6 weeks & HADS-D & $\begin{array}{l}\text { NO significant } \\
\text { difference. }\end{array}$ \\
\hline $\begin{array}{l}\text { Steenbergen et } \\
\text { al. (2015) The } \\
\text { Netherlands }\end{array}$ & Healthy young students & $\begin{array}{l}\text { PRO: } 20 \\
\text { PLA: } 20\end{array}$ & $20 \%$ & $\begin{array}{l}\text { PRO: } \quad 20.2 \quad \pm \quad 2.4 \\
\text { PLA: } 19.7 \pm 1.7\end{array}$ & $\begin{array}{l}\text { Ecologic }{ }^{\circledR} \text { Barrier: B.bifidum } \\
\text { W23, B. lactis W51, B. lactis } \\
\text { W52, L. acidophilus } \\
\text { W37, L. brevis W63, L.casei W56, } \\
\text { L. salivarius W24, L. } \\
\text { lactis }\left(\text { W19 and W58) }\left(2.5 \times 10^{9}\right)\right.\end{array}$ & $\begin{array}{l}\text { Sachet } \\
\text { (powder) }\end{array}$ & 4 weeks & $\begin{array}{l}\text { LEIDs-r; } \\
\text { BDI }\end{array}$ & $\begin{array}{l}\text { NO significant } \\
\text { differences for } \\
\text { both outcomes. }\end{array}$ \\
\hline $\begin{array}{l}\text { Siegel et al. } \\
(2020) \text { USA }\end{array}$ & Healthy young students & $\begin{array}{l}\text { PRO: } \quad 40 \\
\text { PLA: } 39\end{array}$ & $26.55 \%$ & $\begin{array}{l}\text { PRO: } 19.2 \pm 1.1 \\
\text { PLA: } 19.4 \pm 1.0\end{array}$ & B. longum $\left(8 \times 10^{10}\right)$ & Capsules & 1 week & CES-D & $\begin{array}{l}\text { NO significant } \\
\text { difference. }\end{array}$ \\
\hline $\begin{array}{l}\text { Reininghaus } \quad \text { et } \\
\text { al. } \quad(2020) \\
\text { Austria }\end{array}$ & $\begin{array}{l}\text { Adults with MDD treated } \\
\text { with } \\
\text { antidepressant therapy }\end{array}$ & $\begin{array}{l}\text { PRO: } 28 \\
\text { PLA: } 33\end{array}$ & $23.4 \%$ & $\begin{array}{l}\text { PRO: } 43 \pm 14.31 \\
\text { PLA: } 40.11 \pm 11.45\end{array}$ & $\begin{array}{l}\text { B. bifidum W23, B. lactis } \\
\text { W51, B. lactis W52, L. } \\
\text { acidophilus W22, L. casei W56, L. } \\
\text { paracasei W20, L. plantarum } \\
\text { W62, L. salivarius W24 } \\
\text { and L. lactis W19. }\left(7.5 \times 10^{9}\right)\end{array}$ & $\begin{array}{l}\text { Sachet } \\
\text { (powder) }\end{array}$ & 4 weeks & $\begin{array}{l}\text { BDI; } \\
\text { HAMD }\end{array}$ & $\begin{array}{l}\text { NO significant } \\
\text { difference. }\end{array}$ \\
\hline $\begin{array}{l}\text { Moludi et al. } \\
\text { (2019) Iran }\end{array}$ & $\begin{array}{l}\text { Adults with recente MI who } \\
\text { underwent PCI }\end{array}$ & $\begin{array}{l}\text { PRO: } 22 \\
\text { PLA: } 22\end{array}$ & $92.5 \%$ & $\begin{array}{l}\text { PRO: } 56.6 \pm 9.10 \\
\text { PLA: } 57.10 \pm 7.80\end{array}$ & L. rhamnosus $\left(1.6 \times 10^{9}\right)$ & Capsule & 12 weeks & BDI & PRO > PLA. \\
\hline
\end{tabular}


Research, Society and Development, v. 10, n. 9, e54510918359, 2021

(CC BY 4.0) | ISSN 2525-3409 | DOI: http://dx.doi.org/10.33448/rsd-v10i9.18359

\begin{tabular}{|c|c|c|c|c|c|c|c|c|c|}
\hline $\begin{array}{l}\text { Moludi et al. } \\
\text { (2021) Iran }\end{array}$ & Adults with CAD & $\begin{array}{l}\text { PRO: } 24 \\
\text { PLA: } 24\end{array}$ & $56 \%$ & $\begin{array}{l}\text { PRO: } 51.25 \pm 12.66 \\
\text { PLA: } 52.18 \pm 12.78\end{array}$ & $\begin{array}{l}\text { Lactobacillus Rhamnosus } G(1.9 \\
\left.\text { x } 10^{9}\right)\end{array}$ & Capsule & 8 weeks & BDI & PRO > PLA. \\
\hline $\begin{array}{l}\text { Lee } \text { et al. }(2021) \\
\text { Korea }\end{array}$ & $\begin{array}{l}\text { Healthy young students with } \\
\text { halitosis }\end{array}$ & $\begin{array}{l}\text { PRO: } \\
\text { PLA: } 28\end{array}$ & $62.9 \%$ & $\begin{array}{l}\text { PRO: } 23.44 \pm 2.88 \\
\text { PLAS: } 23.75 \pm 3.42\end{array}$ & Weissella cibaria $\mathrm{CMU}\left(8 \times 10^{7}\right)$ & Tablet & 8 weeks & CES-D & $\begin{array}{l}\text { NO significant } \\
\text { difference. }\end{array}$ \\
\hline $\begin{array}{l}\text { Dawe et al. } \\
(2020) \text { New } \\
\text { Zealand }\end{array}$ & $\begin{array}{l}\text { Pregnant Women with } \\
\text { obesity (BMI } \geq 30 \mathrm{~kg} / \mathrm{m} 2)\end{array}$ & $\begin{array}{l}\text { PRO: } \quad 88 \\
\text { PLA: } 76\end{array}$ & 0 & $\begin{array}{l}\text { PRO and PLA:29.39 } \\
\pm 5.39\end{array}$ & $\begin{array}{l}\text { Lactobacillus rhamnosus GG and } \\
\text { Bifdobacterium lactis BB12 }(6.5 x \\
\left.10^{9}\right)\end{array}$ & Capsule & $\begin{array}{l}18-24 \\
\text { weeks }\end{array}$ & EPDS & $\begin{array}{l}\text { NO significant } \\
\text { difference. }\end{array}$ \\
\hline $\begin{array}{l}\text { Chahwan et al. } \\
\text { (2019) Australia }\end{array}$ & $\begin{array}{l}\text { Adults with depressive } \\
\text { symptoms }\end{array}$ & $\begin{array}{l}\text { PRO: } \\
\text { PLA: } 37\end{array}$ & $32,51 \%$ & $\begin{array}{l}\text { PRO: } 36.65 \pm 11.75 \\
\text { PLA: }\end{array}$ & $\begin{array}{l}\text { Ecologic }{ }^{\circledR} \text { Barrier: B. bifdum } \\
\mathrm{W} 23, \text { B. lactis } \mathrm{W} 51 \text { and } \mathrm{W} 52, \text { L. } \\
\text { acidophilus } \mathrm{W} 37 \text {, L. brevis } \mathrm{W} 63 \text {, } \\
\text { L. casei } \mathrm{W} 56, \text { L. salivarius } \mathrm{W} 24 \text {, } \\
\text { Lactococcus } \\
\text { lactis } \mathrm{W} 19 \text { and } \mathrm{W} 58 \\
\left(1 \times 10^{10}\right)\end{array}$ & $\begin{array}{l}\text { Sachet } \\
\text { (powder) }\end{array}$ & 8 week & BDI; BAI & $\begin{array}{l}\text { NO significant } \\
\text { difference. }\end{array}$ \\
\hline $\begin{array}{l}\text { Venkataraman } \\
\text { et al. (2020) } \\
\text { India }\end{array}$ & $\begin{array}{l}\text { Healthy students facing } \\
\text { examination stress }\end{array}$ & $\begin{array}{l}\text { PRO: } \\
\text { PLA: } 38\end{array}$ & 21.25 & $\begin{array}{l}\text { PRO and PLA: } \\
21.4 \pm 1.5\end{array}$ & $\begin{array}{l}\text { (Bacillus coagulans Unique IS2, } \\
\text { L. rhamnosus UBLR58, B. lactis } \\
\text { UBBLa70, L. plantarum UBLP40 } \\
\text { (each of } 2 \text { billion CFU); B. breve } \\
\text { UBBr01, B. infantis UBBI01 ( } 1 \text { x } \\
10^{10} \text { ) }\end{array}$ & Capsule & 4 week & DASS & PRO > PLA. \\
\hline
\end{tabular}

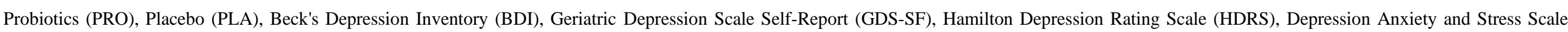

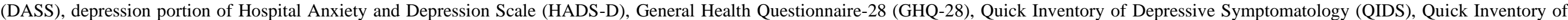

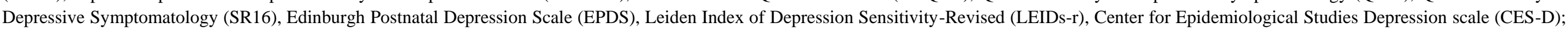
Patient Health Questionnaire 9 (PHT-9); Myocardial Infarction (MI); Percutaneous Coronary Intervention (PCI); Coronary Artery Disease (CAD).

* Numeric results are present in Figure 3

** PRO $>$ PLA were considered when $p<0.05$

Source: Palmares et al., (2021). 
Figure 3: Forest plot showing the standardized mean difference in depressive symptoms, comparing probiotic supplementation and placebo.

\subsection{Major Depressive Disorder population}

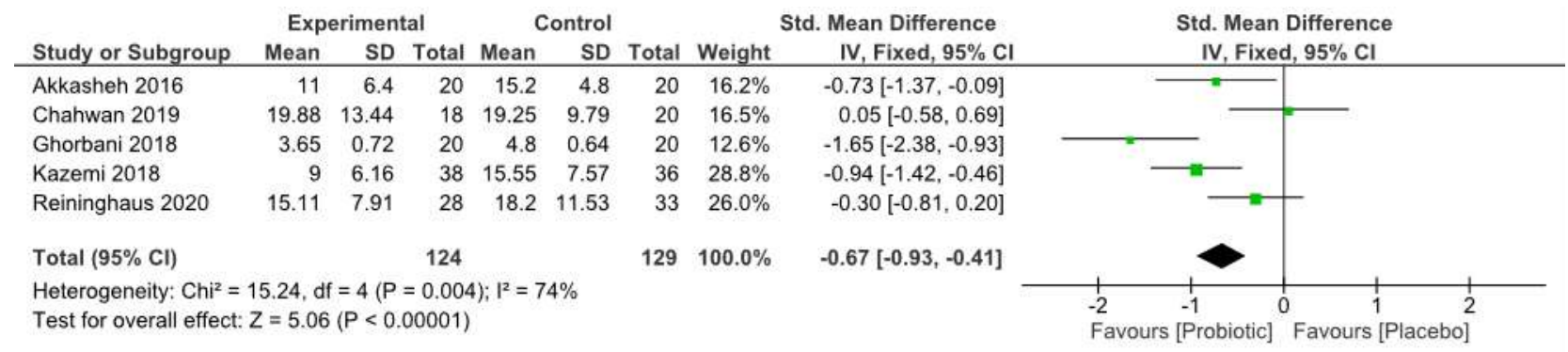

\subsection{Other clinical diagnosis population}

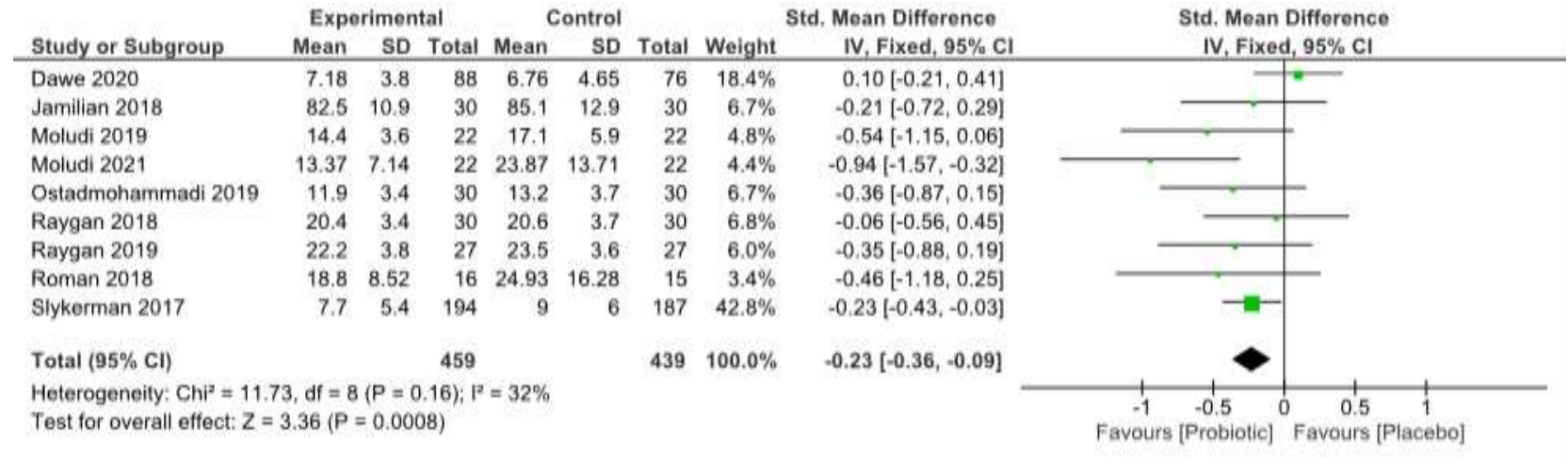

\subsection{Healthy population}

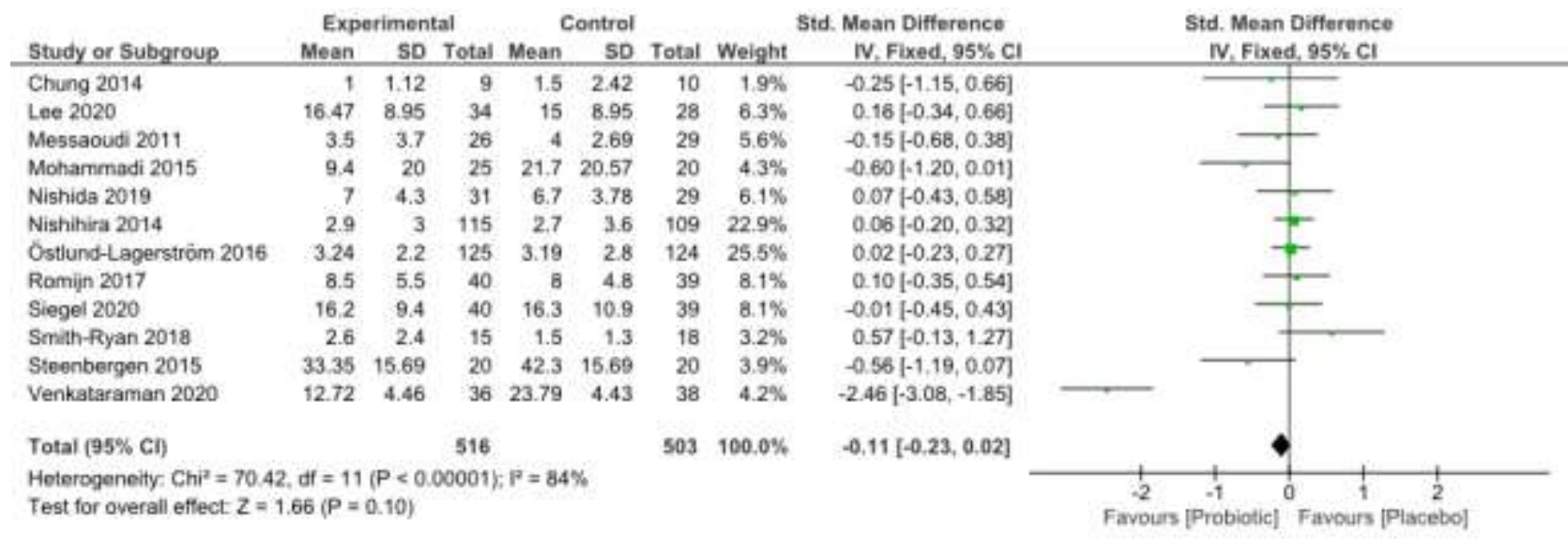

\subsection{Total}

Total $(95 \% \mathrm{Cl})$

1099

Heterogeneity: $\mathrm{Chi}^{2}=112.10, \mathrm{df}=25(\mathrm{P}<0.00001) ; \mathrm{I}^{2}=78 \%$

Test for overall effect: $Z=4.99(P<0.00001)$
$1071 \quad 100.0 \% \quad-0.22[-0.30,-0.13]$

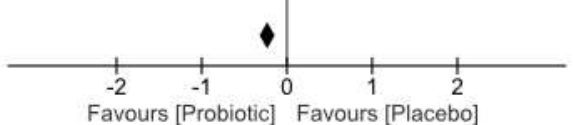

Source: Palmares et al., (2021). 
Figure 4: Funnel plot showing publication bias for depression.

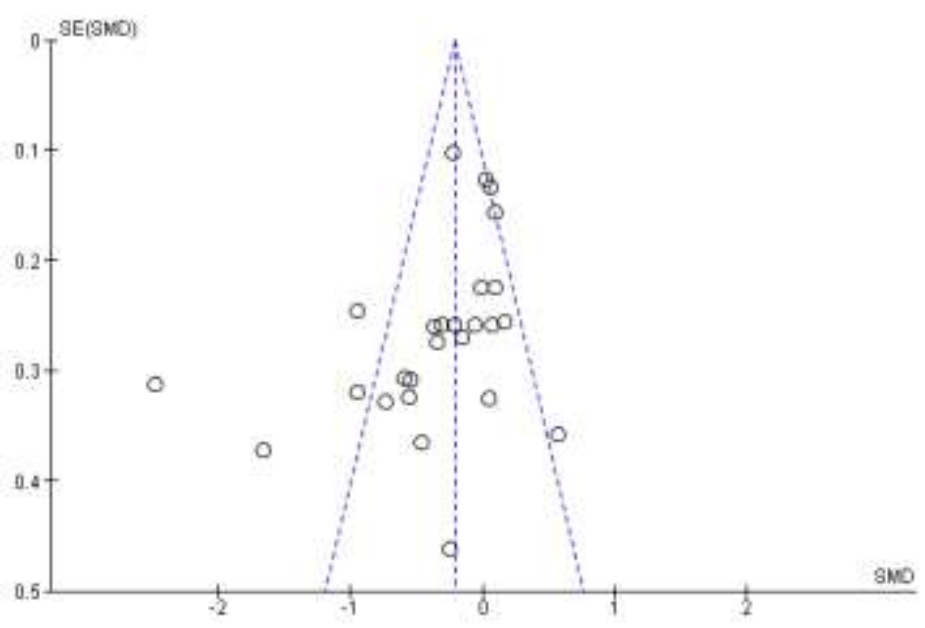

Source: Palmares et al., (2021).

\section{Discussion}

The present meta-analysis suggests that probiotics significantly improved depressive symptoms compared to a placebo. These results differ from those of $\mathrm{Ng}$ et al's meta-analysis ( $\mathrm{Ng}$ et al., 2018), that, in 2018, included 10 articles. In comparison, we included sixteen more studies and increased the number of participants from 1,349 to 2,170 .

Compared to Goh et al's (2019) meta-analysis, we present similar results regarding the positive effect of probiotics on the total population (Figure 3.4), that is, a group of participants that includes healthy people and those diagnosed with depression or other clinical conditions. However, differently from this author, we obtained a result that favors the use of probiotics in participants with other clinical diagnoses (Figure 3.2). The fact that mainly contributes to this difference is that, in the present meta-analysis, studies involving participants with IBS were excluded - Simren et al. (2010) and Pinto-Sanchez et al. (2017) considered that such a condition would represent a potential confounder. According to meta-analyses of Zhang (2016) and Ford (2018), consumption of probiotics significantly improved pain scales and gastrointestinal symptoms in people with IBS. For this reason, it would not be possible to determine to what extent the impact on mood scores would be due to reducing gastrointestinal symptoms.

Similar to antidepressants, probiotics have a greater effect on those diagnosed with MDD. The usually mild depressive symptomatology found in non-depressed individuals may lead to greater dispersion, making it difficult to analyze the effect of interventions, such as probiotics (Goh et al., 2019). Another reason is the commonly found abnormality of the intestinal microbiota in patients with diagnosed depression, which may be a relevant pathophysiological mechanism modified by probiotic use.5 What may explain the effect of probiotics on participants with other clinical conditions is that they tend to have a more depressive baseline mood and a higher prevalence of depression compared to healthy participants (Li et al., 2019). 
It is worth mentioning that, due to the low number of studies, it is difficult to quantify the additional effect of the probiotic in the treatment of depression. Of the five articles with patients diagnosed with MDD eligible for this meta-analysis, only one specified the conventional antidepressant drug used both in the placebo and exposed groups (Ghorbani et al., 2018).

Several mechanisms are suggested to explain the impact of probiotics on mood and are summarized in what is known as the microbiota-gut-brain axis. Preclinical methodology, in comparison to clinical studies, is better suited for studying pathophysiological mechanisms and thus clarifying this axis. Nonetheless, despite optimistic findings in preclinical studies, the use of probiotics in human participants remains a challenge. Models for to assessing changes in behavior and mood in non-human subjects are limited, and their interpretations do not allow large generalizations for humans (Harro, 2019). Animal testing is based on indirect inferences of the animal's mood from objective behavioral data, while human scales are subjective, based on self-rated scales (Harro, 2019). Differences in intestinal microbiota, genetics, and neurological complexity between rodents and humans make the translation of preclinical studies even more difficult for clinical applications (Kelly et al., 2017).

Still, it is known that the vagus nerve has a crucial effect on communication between the intestine and the brain. The administration of probiotics in animals caused behavioral changes related to fear and anxiety, both avoided through the vagotomy prior to the administration of the probiotic (Bercik et al., 2011; Marvel et al., 2004). Short-chain fatty acids, a product of bacterial metabolism, can directly affect animal behavior (DeCastro et al., 2005). Evidence suggests that the intestinal microbiota produces important neurotransmitters or their precursors that, acting locally, can influence the brain through vagus afferences: tryptophans, serotonin, and GABA. Treatment with probiotics can modulate the production of these substances in rodents and humans (Barrett et al., 2012; Desbonnet et al., 2008).

The hypothalamic-pituitary-adrenal (HPA) axis and inflammatory mechanisms have great relevance in the pathophysiology of mood disorders (Foster \& McVey Neufeld, 2013). The use of probiotics reduced the hyperactivity of the HPA axis (Liu et al., 2016), and changes in the intestinal microbiota are related to the proinflammatory state in the brain of rodents and humans (Daulatzai, 2014; Huuskonen et al., 2004). A survey conducted by Mohammadi et al. (2016) demonstrated that the use of probiotics has been able to significantly reduce circulating cortisol levels in humans.

Unlike previous meta-analyses on the topic, asymmetry of the funnel plot was found in this review. Considering the inexistence of inadequate analyses among the RCTs used, as well as the correct choice of the measure of the effect, the pattern found by us suggests publication bias. This indicates a preference for publications in which probiotics demonstrate significant effects. However, since the included RCTs are good methodological quality, this risk of bias is lower.

There is no consensus about strains, the dose in CFU, duration, adjuvants, and frequency of use of probiotics that should be used in humans. The differences in these dosage variables and the depressive mood scales explain the sample's considerable heterogeneity and hence may have interfered with the funnel plot asymmetry. Moreover, most studies showing significative beneficial effect of probiotics came from Iran and all 
studies outside Iran except one from New Zealand and other from India show no beneficial effect. It seems to be a fundamental limitation to generalize the present outcome to other ethnic groups.

Probiotics are notoriously safe treatments with mild and infrequent adverse effects (Niu, 2020). The analyzed studies sporadically indicated nausea (Slykerman et al., 2017), diarrhea, constipation (ÖstlundLagerström et al., 2016), and abdominal discomfort (Roman et al., 2018). Symptoms also present in the placebo group, which may indicate the effect of adjuvant substances (e.g., corn starch, fructooligosaccharides, maltodextrin). This meta-analysis also suggests no significant difference in the dropout rate between the placebo and exposed groups.

One of the most important limitations of this meta-analysis is the lack of uniformity between the designs of the included RCTs. First, the studies involved participants with different diagnoses, with only five involving patients with MDD. Allied to this, a large difference in the methods of therapeutic intervention and the scales to access the results was seen. This contributes to increased heterogeneity and, therefore, limits the power of generalization of the results. Thus, its reduction should be a target in the next meta-analyses. Another limiting fact is that the available studies do not assess the persistence of the effect of the probiotics by long-term follow-up measures. In most studies, only the first postintervention measure, performed immediately after the end of treatment, was presented.

For these reasons, we recommend an elaboration of studies that evaluate the effect of probiotics in patients diagnosed with MDD, with more participants and multiple scales for depressed mood, valuing those that are repeated in the studies. Future research could also ascertain the best dosage of probiotics and compare doses, duration, frequency, formulation, strains, and the number of species as well as the persistence of the effect through follow-up.

\section{Conclusion}

This meta-analysis with 26 RCTs found that probiotic supplementation had a significant effect in reducing depressive symptoms. A subgroup analysis showed a significant reduction in participants with MDD and other diagnosed clinical conditions, but not in healthy volunteers. The methodological differences between the studies and the low number of RCTs involving participants with depression decrease the ability to generalize this meta-analysis. Therefore, we suggest more clinical trials involving a larger number of participants diagnosed with MDD who use probiotics as an adjunct to antidepressants with a focus on using recurrent scales in studies and finding the best dosage.

\section{References}

Akkasheh, G., Kashani-Poor, Z., Tajabadi-Ebrahimi, M., Jafari, P., Akbari, H., Taghizadeh, M., Memarzadeh, M. R., Asemi, Z., \& Esmaillzadeh, A. (2016). Clinical and metabolic response to probiotic administration in patients with major depressive disorder: A randomized, double-blind, placebo-controlled trial. Nutrition, 32(3), 315-320. https://doi.org/10.1016/j.nut.2015.09.003

Astó, E., Méndez, I., Audivert, S., Farran-Codina, A., \& Espadaler, J. (2019). The efficacy of probiotics, prebiotic inulin-type fructans, and synbiotics in human ulcerative colitis: A systematic review and meta-analysis. Nutrients, 11(2), 1-16. https://doi.org/10.3390/nu11020293

Barrett, E., Ross, R. P., O’Toole, P. W., Fitzgerald, G. F., \& Stanton, C. (2012). $\gamma$-Aminobutyric acid production by culturable bacteria from the human intestine. Journal of Applied Microbiology, 113(2), 411-417. https://doi.org/10.1111/j.1365-2672.2012.05344.x

Bercik, P., Park, A. J., Sinclair, D., Khoshdel, A., Lu, J., Huang, X., Deng, Y., Blennerhassett, P. A., Fahnestock, M., Moine, D., Berger, B., Huizinga, J. D., Kunze, W., Mclean, P. G., Bergonzelli, G. E., Collins, S. M., \& Verdu, E. F. (2011). The anxiolytic effect of Bifidobacterium longum NCC3001 involves vagal pathways for gut-brain communication. Neurogastroenterology and Motility, 23(12), 1132-1139. https://doi.org/10.1111/j.1365-2982.2011.01796.x 
Chahwan, B., Kwan, S., Isik, A., van Hemert, S., Burke, C., \& Roberts, L. (2019). Gut feelings: A randomised, triple-blind, placebo-controlled trial of probiotics for depressive symptoms. Journal of Affective Disorders, 253(April), 317-326. https://doi.org/10.1016/j.jad.2019.04.097

Chung, Y. C., Jin, H. M., Cui, Y., Kim, D. S., Jung, J. M., Park, J. Il, Jung, E. S., Choi, E. K., \& Chae, S. W. (2014). Fermented milk of Lactobacillus helveticus IDCC3801 improves cognitive functioning during cognitive fatigue tests in healthy older adults. Journal of Functional Foods, 10 , 465-474. https://doi.org/10.1016/j.jff.2014.07.007

Daulatzai, M. A. (2014). Chronic functional bowel syndrome enhances gut-brain axis dysfunction, neuroinflammation, cognitive impairment, and vulnerability to dementia. Neurochemical Research, 39(4), 624-644. https://doi.org/10.1007/s11064-014-1266-6

Dawe, J. P., McCowan, L. M. E., Wilson, J., Okesene-Gafa, K. A. M., \& Serlachius, A. S. (2020). Probiotics and Maternal Mental Health: A Randomised Controlled Trial among Pregnant Women with Obesity. Scientific Reports, 10(1), 1-11. https://doi.org/10.1038/s41598-020-58129-w

DeCastro, M., Nankova, B. B., Shah, P., Patel, P., Mally, P. V., Mishra, R., \& La Gamma, E. F. (2005). Short chain fatty acids regulate tyrosine hydroxylase gene expression through a cAMP-dependent signaling pathway. Molecular Brain Research, 142(1), 28-38. https://doi.org/10.1016/j.molbrainres.2005.09.002

Desbonnet, L., Garrett, L., Clarke, G., Bienenstock, J., \& Dinan, T. G. (2008). The probiotic Bifidobacteria infantis: An assessment of potential antidepressant properties in the rat. Journal of Psychiatric Research, 43(2), 164-174. https://doi.org/10.1016/j.jpsychires.2008.03.009

Egger, M., Smith, G. D., Schneider, M., \& Minder, C. (1997). Bias in meta-analysis detected by a simple, graphical test. British Medical Journal, 315(7109), 629-634. https://doi.org/10.1136/bmj.315.7109.629

Ford, A. C., Harris, L. A., Lacy, B. E., Quigley, E. M. M., \& Moayyedi, P. (2018). Systematic review with meta-analysis: the efficacy of prebiotics, probiotics, synbiotics and antibiotics in irritable bowel syndrome. Alimentary Pharmacology and Therapeutics, 48(10), 1044-1060. https://doi.org/10.1111/apt.15001

Foster, J. A., \& McVey Neufeld, K. A. (2013). Gut-brain axis: How the microbiome influences anxiety and depression. Trends in Neurosciences, 36(5), 305312. https://doi.org/10.1016/j.tins.2013.01.005

Ghorbani, Z., Nazari, S., Etesam, F., Nourimajd, S., Ahmadpanah, M., \& Razeghi Jahromi, S. (2018). The Effect of Synbiotic as an Adjuvant Therapy to Fluoxetine in Moderate Depression: A Randomized Multicenter Trial. Archives of Neuroscience, 5(2). https://doi.org/10.5812/archneurosci.60507

Goh, K. K., Liu, Y. W., Kuo, P. H., Chung, Y. C. E., Lu, M. L., \& Chen, C. H. (2019). Effect of probiotics on depressive symptoms: A meta-analysis of human studies. Psychiatry Research, 282(September), 112568. https://doi.org/10.1016/j.psychres.2019.112568

Guo, Q., Goldenberg, J. Z., Humphrey, C., El Dib, R., \& Johnston, B. C. (2019). Probiotics for the prevention of pediatric antibiotic-associated diarrhea. Cochrane Database of Systematic Reviews, 2019(4), 1-102. https://doi.org/10.1002/14651858.CD004827.pub5.

Harro, J. (2019). Animal models of depression: pros and cons. Cell and Tissue Research, 377(1), 5-20. https://doi.org/10.1007/s00441-018-2973-0

Higgins, J. P. T., Altman, D. G., Gøtzsche, P. C., Jüni, P., Moher, D., Oxman, A. D., Savović, J., Schulz, K. F., Weeks, L., \& Sterne, J. A. C. (2011). The Cochrane Collaboration's tool for assessing risk of bias in randomised trials. BMJ (Online), 343(7829), 1-9. https://doi.org/10.1136/bmj.d5928

Huuskonen, J., Suuronen, T., Nuutinen, T., Kyrylenko, S., \& Salminen, A. (2004). Regulation of microglial inflammatory response by sodium butyrate and short-chain fatty acids. British Journal of Pharmacology, 141(5), 874-880. https://doi.org/10.1038/sj.bjp.0705682

Jamilian, M., Mansury, S., Bahmani, F., Heidar, Z., Amirani, E., \& Asemi, Z. (2018). The effects of probiotic and selenium co-supplementation on parameters of mental health, hormonal profiles, and biomarkers of inflammation and oxidative stress in women with polycystic ovary syndrome 11 Medical and Health Sciences 1103 Clinical Sciences. Journal of Ovarian Research, 11(1), 1-7. https://doi.org/10.1186/s13048-018-0457-1

Jiang, H., Ling, Z., Zhang, Y., Mao, H., Ma, Z., Yin, Y., Wang, W., Tang, W., Tan, Z., Shi, J., Li, L., \& Ruan, B. (2015). Altered fecal microbiota composition in patients with major depressive disorder. Brain, behaviour, and immunity, 48(April), 186-194. https://doi.org/10.1016/j.bbi.2015.03.016

Kazemi, A., Noorbala, A. A., Azam, K., Eskandari, M. H., \& Djafarian, K. (2019). Effect of probiotic and prebiotic vs placebo on psychological outcomes in patients with major depressive disorder: A randomized clinical trial. Clinical Nutrition, 38(2), 522-528. https://doi.org/10.1016/j.clnu.2018.04.010

Kelly, J. R., Allen, A. P., Temko, A., Hutch, W., Kennedy, P. J., Farid, N., Murphy, E., Boylan, G., Bienenstock, J., Cryan, J. F., Clarke, G., \& Dinan, T. G. (2017). Lost in translation? The potential psychobiotic Lactobacillus rhamnosus (JB-1) fails to modulate stress or cognitive performance in healthy male subjects. Brain, Behavior, and Immunity, 61, 50-59. https://doi.org/10.1016/j.bbi.2016.11.018

Kong, X., Liu, J., Cetinbas, M., Sadreyev, R., Koh, M., Liu, K., \& Onderdonk, A. B. (2019). New and Preliminary Evidence on Altered Oral and Disorder (ASD ): Implications for ASD Diagnosis and. Nutrients, 11, 1-26. https://doi.org/10.3390/nu11092128

Kupfer, D. J., \& Regier, D. A. (2014). Diagnostic and Statistical Manual of Mental Disorders, Fifth Edition.

Lee, D. S., Kim, M., Nam, S. H., Kang, M. S., \& Lee, S. A. (2021). Effects of oral probiotics on subjective halitosis, oral health, and psychosocial health of college students: A randomized, double-blind, placebo-controlled study. International Journal of Environmental Research and Public Health, 18(3), 1-10. https://doi.org/10.3390/ijerph18031143

Li, H., Ge, S., Greene, B., \& Dunbar-Jacob, J. (2019). Depression in the context of chronic diseases in the United States and China. International Journal of Nursing Sciences, 6(1), 117-122. https://doi.org/10.1016/j.ijnss.2018.11.007

Liberati, A., Altman, D. G., Tetzlaff, J., Mulrow, C., Gøtzsche, P. C., Ioannidis, J. P. A., Clarke, M., Devereaux, P. J., Kleijnen, J., \& Moher, D. (2009). The PRISMA statement for reporting systematic reviews and meta-analyses of studies that evaluate health care interventions: Explanation and elaboration. PLoS Medicine, 6(7), 1-28. https://doi.org/10.1371/journal.pmed.1000100 
Liu, Y. W., Liu, W. H., Wu, C. C., Juan, Y. C., Wu, Y. C., Tsai, H. P., Wang, S., \& Tsai, Y. C. (2016). Psychotropic effects of Lactobacillus plantarum PS128 in early life-stressed and naïve adult mice. Brain Research, 1631, 1-12. https://doi.org/10.1016/j.brainres.2015.11.018

Ma, Z. F., Yusof, N., Hamid, N., Lawenko, R. M., Wan Mohammad, W. M. Z., Liong, M. T., Sugahara, H., Odamaki, T., Xiao, J., \& Lee, Y. Y. (2019). Bifidobacterium infantis M-63 improves mental health in victims with irritable bowel syndrome developed after a major flood disaster. Beneficial Microbes, 10(2), 111-120. https://doi.org/10.3920/BM2018.0008

Marvel, F. A., Chen, C. C., Badr, N., Gaykema, R. P. A., \& Goehler, L. E. (2004). Reversible inactivation of the dorsal vagal complex blocks lipopolysaccharide-induced social withdrawal and c-Fos expression in central autonomic nuclei. Brain, Behavior, and Immunity, 18(2), 123-134. https://doi.org/10.1016/j.bbi.2003.09.004

McCormack, J., \& Korownyk, C. (2018). Effectiveness of antidepressants. BMJ (Online), 360, 9-10. https://doi.org/10.1136/bmj.k1073

Messaoudi, M., Lalonde, R., Violle, N., Javelot, H., Desor, D., Nejdi, A., Bisson, J. F., Rougeot, C., Pichelin, M., Cazaubiel, M., \& Cazaubiel, J. M. (2011). Assessment of psychotropic-like properties of a probiotic formulation (Lactobacillus helveticus R0052 and Bifidobacterium longum R0175) in rats and human subjects. British Journal of Nutrition, 105(5), 755-764. https://doi.org/10.1017/S0007114510004319

Mohammadi, A. A., Jazayeri, S., Khosravi-Darani, K., Solati, Z., Mohammadpour, N., Asemi, Z., Adab, Z., Djalali, M., Tehrani-Doost, M., Hosseini, M., \& Eghtesadi, S. (2016). The effects of probiotics on mental health and hypothalamic-pituitary-adrenal axis: A randomized, double-blind, placebo-controlled trial in petrochemical workers. Nutritional Neuroscience, 19(9), 387-395. https://doi.org/10.1179/1476830515Y.0000000023

Moher, D., Liberati, A., Tetzlaff, J., Altman, D. G., Altman, D., Antes, G., Atkins, D., Barbour, V., Barrowman, N., Berlin, J. A., Clark, J., Clarke, M., Cook, D., D’Amico, R., Deeks, J. J., Devereaux, P. J., Dickersin, K., Egger, M., Ernst, E., ... Tugwell, P. (2009). Preferred reporting items for systematic reviews and meta-analyses: The PRISMA statement. PLoS Medicine, 6(7), 1-7. https://doi.org/10.1371/journal.pmed.1000097

Moludi, J., Alizadeh, M., Mohammadzad, M. H. S., \& Davari, M. (2019). The Effect of Probiotic Supplementation on Depressive Symptoms and Quality of Life in Patients after Myocardial Infarction: Results of a Preliminary Double-Blind Clinical Trial. Psychosomatic Medicine, 81(9), 770-777. https://doi.org/10.1097/PSY.0000000000000749

Moludi, J., Khedmatgozar, H., Nachvak, S. M., Abdollahzad, H., Moradinazar, M., \& Sadeghpour tabaei, A. (2021). The effects of co-administration of probiotics and prebiotics on chronic inflammation, and depression symptoms in patients with coronary artery diseases: a randomized clinical trial. Nutritional Neuroscience, 0(0), 1-10. https://doi.org/10.1080/1028415X.2021.1889451

Myers, B., \& Greenwood-Van Meerveld, B. (2009). Role of anxiety in the pathophysiology of irritable bowel syndrome: Importance of the amygdala. Frontiers in Neuroscience, 3(47), 1-10. https://doi.org/10.3389/neuro.21.002.2009

Ng, Q. X., Peters, C., Ho, C. Y. X., Lim, D. Y., \& Yeo, W. S. (2018). A meta-analysis of the use of probiotics to alleviate depressive symptoms. Journal of Affective Disorders, 228, 13-19. https://doi.org/10.1016/j.jad.2017.11.063

Nishida, K., Sawada, D., Kuwano, Y., Tanaka, H., \& Rokutan, K. (2019). Health benefits of lactobacillus gasseri cp2305 tablets in young adults exposed to chronic stress: A randomized, double-blind, placebo-controlled study. Nutrients, 11(8). https://doi.org/10.3390/nu11081859

Nishihira, J., Kagami-Katsuyama, H., Tanaka, A., Nishimura, M., Kobayashi, T., \& Kawasaki, Y. (2014). Elevation of natural killer cell activity and alleviation of mental stress by the consumption of yogurt containing Lactobacillus gasseri SBT2055 and Bifidobacterium longum SBT2928 in a double-blind, placebo-controlled clinical trial. Journal of Functional Foods, 11(C), 261-268. https://doi.org/10.1016/j.jff.2014.09.002

Niu, H. L., \& Xiao, J. Y. (2020). The efficacy and safety of probiotics in patients with irritable bowel syndrome: Evidence based on 35 randomized controlled trials. International Journal of Surgery, 75(80), 116-127. https://doi.org/10.1016/j.ijsu.2020.01.142

Ostadmohammadi, V., Jamilian, M., Bahmani, F., \& Asemi, Z. (2019). Vitamin D and probiotic co-supplementation affects mental health, hormonal, inflammatory and oxidative stress parameters in women with polycystic ovary syndrome. Journal of Ovarian Research, 12(1), 1-8. https://doi.org/10.1186/s13048-019-0480-x

Östlund-Lagerström, L., Kihlgren, A., Repsilber, D., Björkstén, B., Brummer, R. J., \& Schoultz, I. (2016). Probiotic administration among free-living older adults: A double blinded, randomized, placebo-controlled clinical trial. Nutrition Journal, 15(1), 1-10. https://doi.org/10.1186/s12937-016-0198-1

Petra, A. I., Panagiotidou, S., Hatziagelaki, E., Stewart, J. M., Conti, P., \& Theoharides, T. C. (2015). Gut-Microbiota-Brain Axis and Its Effect on Neuropsychiatric Disorders with Suspected Immune Dysregulation. Clinical Therapeutics, 37(5), 984-995. https://doi.org/10.1016/j.clinthera.2015.04.002

Pinto-Sanchez, M. I., Hall, G. B., Ghajar, K., Nardelli, A., Bolino, C., Lau, J. T., Martin, F. P., Cominetti, O., Welsh, C., Rieder, A., Traynor, J., Gregory, C., De Palma, G., Pigrau, M., Ford, A. C., Macri, J., Berger, B., Bergonzelli, G., Surette, M. G., ... Bercik, P. (2017). Probiotic Bifidobacterium longum NCC3001 Reduces Depression Scores and Alters Brain Activity: A Pilot Study in Patients With Irritable Bowel Syndrome. Gastroenterology, 153(2), 448459.e8. https://doi.org/10.1053/j.gastro.2017.05.003

Raygan, F., Ostadmohammadi, V., \& Asemi, Z. (2019). The effects of probiotic and selenium co-supplementation on mental health parameters and metabolic profiles in type 2 diabetic patients with coronary heart disease: A randomized, double-blind, placebo-controlled trial. Clinical Nutrition, 38(4), 1594-1598. https://doi.org/10.1016/j.clnu.2018.07.017

Raygan, F., Ostadmohammadi, V., Bahmani, F., \& Asemi, Z. (2018). The effects of vitamin D and probiotic co-supplementation on mental health parameters and metabolic status in type 2 diabetic patients with coronary heart disease: A randomized, double-blind, placebo-controlled trial. Progress in NeuroPsychopharmacology and Biological Psychiatry, 84, 50-55. https://doi.org/10.1016/j.pnpbp.2018.02.007

Reininghaus, E. Z., Platzer, M., Kohlhammer-Dohr, A., Hamm, C., Mörkl, S., Bengesser, S. A., Fellendorf, F. T., Lahousen-Luxenberger, T., Leitner-Afschar, B., Schöggl, H., Amberger-Otti, D., Wurm, W., Queissner, R., Birner, A., Falzberger, V. S., Painold, A., Fitz, W., Brunnmayr, M., Rieger, A., ... Dalkner, N. (2020). Provit: Supplementary probiotic treatment and vitamin b7 in depression-a randomized controlled trial. Nutrients, 12(11), 1-17. https://doi.org/10.3390/nu12113422 
Research, Society and Development, v. 10, n. 9, e54510918359, 2021

(CC BY 4.0) | ISSN 2525-3409 | DOI: http://dx.doi.org/10.33448/rsd-v10i9.18359

Roman, P., Estévez, A. F., Miras, A., Sánchez-Labraca, N., Cañadas, F., Vivas, A. B., \& Cardona, D. (2018). A Pilot Randomized Controlled Trial to Explore Cognitive and Emotional Effects of Probiotics in Fibromyalgia. Scientific Reports, 8(1), 1-9. https://doi.org/10.1038/s41598-018-29388-5

Romijn, A. R., Rucklidge, J. J., Kuijer, R. G., \& Frampton, C. (2017). A double-blind, randomized, placebo-controlled trial of Lactobacillus helveticus and Bifidobacterium longum for the symptoms of depression. Australian and New Zealand Journal of Psychiatry, 51(8), 810-821. https://doi.org/10.1177/0004867416686694

Sender, R., Fuchs, S., \& Milo, R. (2016). Revised Estimates for the Number of Human and Bacteria Cells in the Body. PLoS Biology, 14(8), 1-14. https://doi.org/10.1371/journal.pbio. 1002533

Sherwin, E., Sandhu, K. V., Dinan, T. G., \& Cryan, J. F. (2016). May the Force Be With You: The Light and Dark Sides of the Microbiota-Gut-Brain Axis in Neuropsychiatry. CNS Drugs, 30(11), 1019-1041. https://doi.org/10.1007/s40263-016-0370-3

Siegel, M. P., \& Conklin, S. M. (2020). Acute intake of B. longum probiotic does not reduce stress, anxiety, or depression in young adults: A pilot study. Brain, Behavior, \& Immunity - Health, 2(January), 100029. https://doi.org/10.1016/j.bbih.2019.100029

Simrén, M., Öhman, L., Olsson, J., Svensson, U., Ohlson, K., Posserud, I., \& Strid, H. (2010). Clinical trial: The effects of a fermented milk containing three probiotic bacteria in patients with irritable bowel syndrome - A randomized, double-blind, controlled study. Alimentary Pharmacology and Therapeutics, 31(2), 218-227. https://doi.org/10.1111/j.1365-2036.2009.04183.x

Slykerman, R. F., Hood, F., Wickens, K., Thompson, J. M. D., Barthow, C., Murphy, R., Kang, J., Rowden, J., Stone, P., Crane, J., Stanley, T., Abels, P., Purdie, G., Maude, R., \& Mitchell, E. A. (2017). Effect of Lactobacillus rhamnosus HN001 in Pregnancy on Postpartum Symptoms of Depression and Anxiety: A Randomised Double-blind Placebo-controlled Trial. EBioMedicine, 24, 159-165. https://doi.org/10.1016/j.ebiom.2017.09.013

Smith-ryan, A. E., Mock, M. G., Trexler, E. T., Hirsch, K. R., \& Blue, M. N. M. (n.d.). Influence of a multi-strain probiotic on body composition and mood in female occupational shift-workers 1 Applied Physiology Laboratory, Department of Exercise and Sport Science , The University of North Carolina , Chapel Hill, NC,27599; meremock@gma.1-36.

Stasi, C., Caserta, A., Nisita, C., Cortopassi, S., Fani, B., Salvadori, S., Pancetti, A., Bertani, L., Gambaccini, D., de Bortoli, N., Dell'Osso, L., Blandizzi, C., Marchi, S., \& Bellini, M. (2019). The complex interplay between gastrointestinal and psychiatric symptoms in irritable bowel syndrome: A longitudinal assessment. Journal of Gastroenterology and Hepatology (Australia), 34(4), 713-719. https://doi.org/10.1111/jgh.14375

Steenbergen, L., Sellaro, R., van Hemert, S., Bosch, J. A., \& Colzato, L. S. (2015). A randomized controlled trial to test the effect of multispecies probiotics on cognitive reactivity to sad mood. Brain, Behavior, and Immunity, 48, 258-264. https://doi.org/10.1016/j.bbi.2015.04.003

Venkataraman, R., Madempudi, R. S., Neelamraju, J., Ahire, J. J., Vinay, H. R., Lal, A., Thomas, G., \& Stephen, S. (2021). Effect of Multi-strain Probiotic Formulation on Students Facing Examination Stress: a Double-Blind, Placebo-Controlled Study. Probiotics and Antimicrobial Proteins, 13(1), 12-18. https://doi.org/10.1007/s12602-020-09681-4

World Health Organization. (2001). The World Health Report 2001.

World Health Organization. (2017). Depression and Other Common Mental Disorders. In Global Health Estimates (pp. 3-20). https://apps.who.int/iris/bitstream/handle/10665/254610/WHO-MSD-MER-2017.2eng.pdf;jsessionid=E00B1CEF3F2485F726AC7B255BEB4758? sequence $=1$

Zhang, Y., Li, L., Guo, C., Mu, D., Feng, B., Zuo, X., \& Li, Y. (2016). Effects of probiotic type, dose and treatment duration on irritable bowel syndrome diagnosed by Rome III criteria: A meta-analysis. BMC Gastroenterology, 16(1), 1-11. https://doi.org/10.1186/s12876-016-0470-Z 